\title{
Peroxiredoxin 5 (PRDX5) is differentially expressed in the brains of patients with
} psychotic disorders.

Shahan Mamoor ${ }^{1}$

1shahanmamoor@gmail.com

East Islip, NY 11730

We used public and published microarray data1,2 to identify the most significant gene expression changes in the brains of patients with psychotic disorders. We identified PRDX53 as differentially expressed in the dorsolateral prefrontal cortex of patients with schizophrenia as well as in the parvalbumin-positive layer 3 neurons of the dorsolateral prefrontal cortex of patients with schizophrenia and schizoaffective disorder. The brain tissues of patients with psychotic disorders expressed significantly lower levels of PRDX5 than that of non-affected control subjects. PRDX5 may be relevant to the biology of schizophrenia and related psychotio disorders.

Keywords: schizophrenia, psychotic disorders, systems biology of schizophrenia, psychosis, PRDX5. 
Psychotic disorders including schizophrenia and schizoaffective disorder are characterized by the presence of psychosis, or an inability to engage in reality testing ${ }^{4,5}$ Patients with psychotic disorders can experience positive as well as negative symptoms ${ }^{6}$ Positive symptoms include hallucinations, visual and auditory, as well as delusions, or fixec false beliefs 6 . Negative symptoms include anhedonia, or an inability to experience pleasure, as well as avolition, or loss of motivation ${ }^{6}$. To understand how specific regions of the brain differ most significantly in terms of gene expression in patients with psychotic disorders, we used published and public microarray data to compare global gene expression profiles of brain tissues from patients with psychotic disorders with that of non-affected, control subjects ${ }^{1,2}$ We found that PRDX5 was among the genes whose expression changed most significantly in patients with schizophrenia in the dorsolateral prefrontal cortex, as well as in the layer 3 parvalbumin-positive neurons of the dorsolateral prefrontal cortex in patients with schizophrenia and schizoaffective disorder. PRDX5 may be relevant for future study of genes pertinent to the biology of psychotic disorders.

\section{Methods}

We used microarray datasets GSE87610 1 and GSE935772 for this differential gene expression analysis in conjunction with GEO2R. GSE87610 was generated using Affymetrix Human Genome U219 with $n=72$ for control subjects and $n=65$ for patients with schizophrenia GSE93577 was generated using Affymetrix Human Genome U219 Array technology with $n=68$ for control subjects and $n=70$ for patients with schizophrenia and schizoaffective disorders. The Benjamini and Hochberg method of $p$-value adjustment was used for ranking of differentia expression but raw p-values were used for assessment of statistical significance of globa differential expression. Log-transformation of data was auto-detected, and the NCB generated category of platform annotation was used. A statistical test was performed to evaluate whether PRDX5 expression was significantly different when comparing normal DLPFO 2 OF 11 
tissue from control subjects and DLPFC or L3 PV+ neurons of the DLPFC from patients with schizophrenia or schizophrenia and schizoaffective disorder, respectively, using a two-tailed unpaired t-test with Welch's correction. Only $\mathrm{p}$-values less than 0.05 were considered statistically significant. We used PRISM for all statistical analyses (Version 8.4.0)(455).

\section{Results}

We mined microarray datasets, public ${ }^{1}$ and published $^{2}$, to identify genes whose expression was most different in the brains of patients with psychotic disorders when compared to control subjects.

PRDX5 is differentially expressed in the dorsolateral prefrontal cortex of patients with schizophrenia.

We identified PRDX5 as among the genes whose mRNA expression was most different in the dorsolateral prefrontal cortex of patients with schizophrenia when compared to contro subjects ${ }^{1}$. When sorting all of the genes expressed in the dorsolateral prefrontal cortex, as measured by microarray, based on change in expression between psychotic patients and control subjects, PRDX5 ranked 8 out of 12188 total transcripts. Differential expression of PRDX5 in the dorsolateral prefrontal cortex of patients with schizophrenia was statistically significant (Table 1; $\mathrm{p}=5.09 \mathrm{E}-09)$.

PRDX5 is differentially expressed in the PV+ L3 neurons dorsolateral prefrontal cortex of patients with schizophrenia and schizoaffective disorder.

In a separate dataset, we identified PRDX5 as differentially expressed in the parvalbumin (PV)-positive L3 neurons of the dorsolateral prefrontal cortex of patients with schizophrenia or schizoaffective disorder when compared to control subjects ${ }^{2}$. When sorting all of the genes expressed in PV+ L3 neurons of the dorsolateral prefrontal cortex, as measured by microarray, based on change in expression between patients with schizophrenia or schizoaffective disorder and control subjects, PRDX5 ranked 4 out of 49386 total transcripts. 
Differential expression of PRDX5 in the PV+ L3 neurons of the DLPFC of patients with schizophrenia or schizoaffective disorder was statistically significant (Table $2 ; p=1.59 E-07$ ).

PRDX5 is expression is significantly lower in the dorsolateral prefrontal cortex of patients with schizophrenia.

Next we obtained exact mRNA expression levels of PRDX5 from the dorsolateral prefrontal cortex of patients with schizophrenia and from the dorsolateral prefrontal cortex of control subjects to directly compare PRDX5 expression between these groups. We also performed a statistical test to evaluate whether this difference in expression was statistically significant. PRDX5 was expressed at significantly lower levels in the dorsolateral prefrontal cortex of patients with schizophrenia when compared to control subjects (Figure 1; $p<0.0001$ ). We calculated a $0.9623 \pm 0.0380$ fold change in PRDX5 expression in the dorsolateral prefrontal cortex of patients with schizophrenia when compared to control subjects (Table 1).

PRDX5 is expression is significantly lower in the PV+ L3 neurons dorsolateral prefrontal cortex of patients with schizophrenia and schizoaffective disorder.

We also obtained exact mRNA expression levels of PRDX5 from PV+ L3 neurons of the dorsolateral prefrontal cortex from patients with schizophrenia or schizoaffective disorder and from the dorsolateral prefrontal cortex of control subjects to directly compare PRDX5 between these two groups. We also performed a statistical test to evaluate whether this difference in expression was statistically significant. PRDX5 was expressed at significantly lower levels in the PV+ L3 neurons of the dorsolateral prefrontal cortex of patients with schizophrenia or schizoaffective disorder when compared to control subjects (Figure $2 ; p<0.0001$ ). We calculated a $0.930 \pm 0.091$ fold change in PRDX5 expression in the PV+ L3 neurons of the dorsolateral prefrontal cortex of patients with schizophrenia and schizoaffective disorder when compared to control subjects (Table 2). 
Thus, we found using public and published microarray datasets 2,3 that PRDX5 was among the genes most differentially expressed in the brain tissues of patients with psychotic disorders, in the dorsolateral prefrontal cortex and in the PV+ $\mathrm{L} 3$ neurons of the dorsolateral prefrontal cortex.

\section{Discussion}

We compared transcriptome information from published ${ }^{1}$ and public $^{2}$ microarray datasets generated using the brain tissues of patients with psychotic disorders, specifically, in the dorsolateral prefrontal cortex, to that of non-affected control subjects, to identify the mos significant changes in gene expression in the brains of patients with psychotic disorders Changes in PRDX5 gene expression were more significant than greater than $99 \%$ of transcriptome in both groups of patients.

PRDX5/PrxV is a member of a family of periredoxins that protect tissue from nitrooxidative damage by reducing peroxides containing a "peroxidatic" cysteine $\left(C_{P}\right)$ residue reactive oxygen and reactive nitrogen species $^{3}$. There are six mammalian Prx isoforms classified as 2-Cys, atypical 2-Cys, and 1-Cys based on the presence and location of the $C_{R}$ PRDX5 is classified as an atypical 2-Cys peroxidoxin ${ }^{3}$. $\mathrm{CP}_{\mathrm{P}} \mathrm{SOH}$ is oxidized to cysteine sulfenid acid ( $\left.\mathrm{C}_{\mathrm{P}} \mathrm{SOH}\right)$. The $\mathrm{C}_{\mathrm{P}}-\mathrm{SOH}$ moiety then reacts with the "resolving" cysteine residue, $\mathrm{C}_{\mathrm{R}}-\mathrm{SH}$, to generate an intrasubunit disulfide ${ }^{3}$.

PRDX5 displayed neuronal-specific expression unlike glial expression of PRDX1 PRDX4 and PRDX6. PRDX5 is expressed in the olfactory bulb, the cerebral cortex, the pons nuclei, the red nucleus, cranial nerve nuclei, the cerebellum, and in motor neurons of the spina cord, whereas PRDX5 expression was relatively low in the CA1/2 pyramidal cells of the hippocampus and in the dopaminergic neurons of the substantia nigra7. In a mouse model of excitotoxic challenge-induced brain lesion, PRDX5 could protect against excitotoxic stress by ibotenate (acting at NMDA receptors) but not by that induced by bromowillardiine (acting a 
AMPA-kainate receptors) ${ }^{8}$. Administration of recombinant mutant PRDX5 that lacks peroxidase activity failed to protect against ibotenate challenge, demonstrating that the antioxidant activity of PRDX5 was important for its neuroprotective properties in the presence of NMDA-induced excitotoxicity ${ }^{8}$.

Identifying the most significant transcriptional features of the brains of patients with psychotic disorders based on quantitative change compared to the normal brain using published and public microarray data identified PRDX5 as a differentially expressed gene and revealed that PRDX5 expression was significantly higher levels in the dorsolateral prefrontal cortex of patients with psychotic disorders. 


\section{References}

1. Arion, D., Huo, Z., Enwright, J.F., Corradi, J.P., Tseng, G. and Lewis, D.A., 2017. Transcriptome alterations in prefrontal pyramidal cells distinguish schizophrenia from bipolar and major depressive disorders. Biological psychiatry, 82(8), pp.594-600.

2. GSE93577. Enwright J.F., Huo Z, Arion D, Corradi J.P, Tseng G., and Lewis D.A. University of Pittsburgh, Psychiatry and Neuroscience. Pittsburgh, PA. https://www.ncbi.nlm.nih.gov geo/query/acc.cgi?acc=GSE93577

3. Knoops, B., Goemaere, J., Van der Eecken, V. and Declercq, J.P., 2011. Peroxiredoxin 5: structure, mechanism, and function of the mammalian atypical 2-Cys peroxiredoxin. Antioxidants \& redox signaling, 15(3), pp.817-829.

4. Cannon, T.D., 2015. How schizophrenia develops: cognitive and brain mechanisms underlying onset of psychosis. Trends in cognitive sciences, 19(12), pp.744-756.

5. Arciniegas, D.B., 2015. Psychosis. Continuum: Lifelong Learning in Neurology, 21(3 Behavioral Neurology and Neuropsychiatry), p.715.

6. Andreasen, N.C., Nopoulos, P., Schultz, S., Miller, D., Gupta, S., Swayze, V. and Flaum, M., 1994. Positive and negative symptoms of schizophrenia: past, present, and future. Acta Psychiatrica Scandinavica, 90, pp.51-59.

7. Goemaere, J. and Knoops, B., 2012. Peroxiredoxin distribution in the mouse brain with emphasis on neuronal populations affected in neurodegenerative disorders. Journal of Comparative Neurology, 520(2), pp.258-280.

8. Plaisant, F., Clippe, A., Vander Stricht, D., Knoops, B. and Gressens, P., 2003. Recombinant peroxiredoxin 5 protects against excitotoxic brain lesions in newborn mice. Free Radical Biology and Medicine, 34(7), pp.862-872. 
Table 1: PRDX5 is differentially expressed in the dorsolateral prefrontal cortex of patients with schizophrenia when compared to control subjects.

The rank of differential expression, the probe/transcript ID, the p-value with respect to global differential expression, $t$, a moderated t-statistic, $B$, the log-odds of differential expression between the two groups compared, the fold change of PRDX5 in the patient population when compared to control subjects, the gene and gene name are listed in this chart. 
Table 2: PRDX5 is differentially expressed in PV+ L3 neurons of the dorsolateral prefrontal cortex of patients with schizophrenia or schizoaffective disorder when compared to control subjects.

The rank of differential expression, the probe/transcript ID, the $p$-value with respect to global differential expression, $t$, a moderated t-statistic, $B$, the log-odds of differential expression between the two groups compared, the fold change of PRDX5 in the patient population when compared to control subjects, the gene and gene name are listed in this chart.

\begin{tabular}{|r|l|l|l|l|l|l|} 
Rank & ID & p-value & t & B & Fold change & Gene \\
\hline 4 & $11717429 \_x \_a t$ & $1.59 E-07$ & -5.5171012 & 6.842591 & $0.930 \pm$ & PRDX5 \\
& & & & & 0.091 & \\
\hline
\end{tabular}




\section{PRDX5}

dorsolateral prefrontal cortex

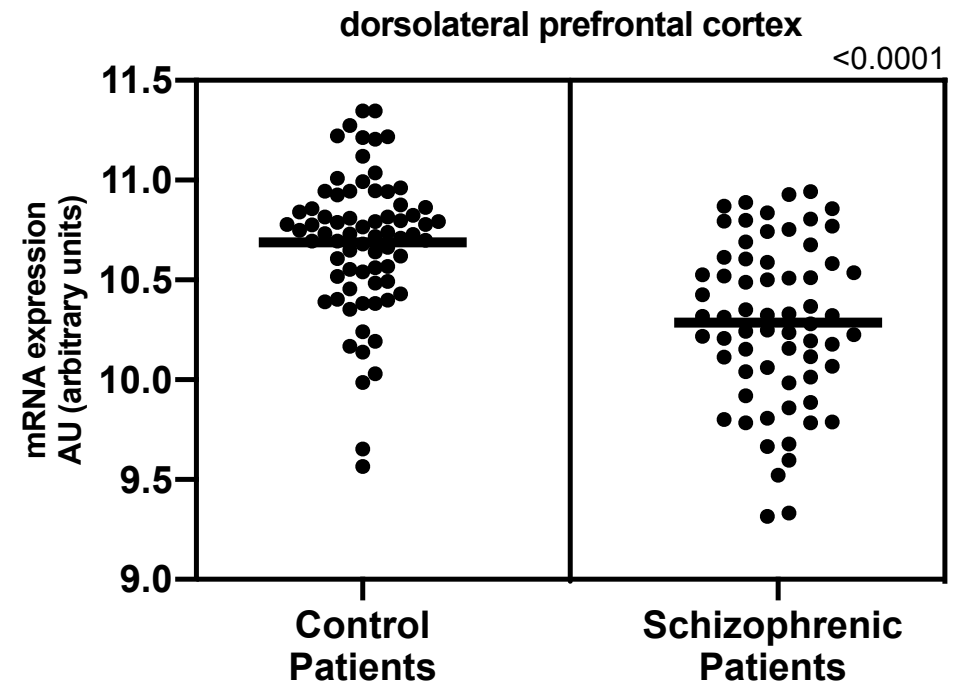

Figure 1: PRDX5 is expressed at significantly lower levels in the dorsolateral prefrontal cortex of patients with schizophrenia when compared to control subjects.

The mRNA expression of PRDX5 in the dorsolateral prefrontal cortex of control subjects (left) and in the dorsolateral prefrontal cortex of patients with schizophrenia (right) is represented with mean mRNA expression level marked and the result of a statistical test evaluating the significance of difference in PRDX5 expression between the dorsolateral prefrontal cortex of control subjects and patients with schizophrenia, a p-value, listed above. 


\section{PRDX5}

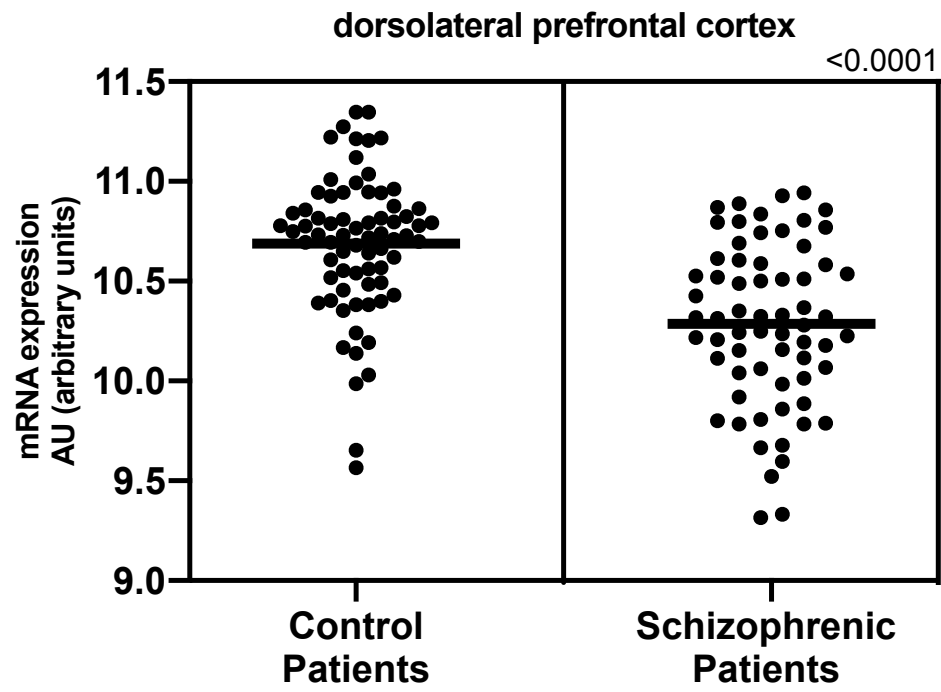

Figure 2: PRDX5 is expressed at significantly lower levels in the PV+ L3 neurons of the dorsolateral prefrontal cortex of patients with schizophrenia or schizoaffective disorder than in the PV+ L3 neurons of the DLPFC of control subjects.

The mRNA expression of PRDX5 in the PV+ L3 neurons of the dorsolateral prefrontal cortex of control subjects (left) and in the PV+ L3 neurons of the dorsolateral prefrontal cortex of patients with schizophrenia or schizoaffective disorder (right) is represented with mean mRNA expression level marked and the result of a statistical test evaluating the significance of difference in PRDX5 expression between the PV+ L3 neurons of the dorsolateral prefrontal cortex of control subjects and patients with schizophrenia or schizoaffective disorder, a pvalue, listed above. 